\title{
Ion-induced Transformation of Metastable Phases
}

Lucille Giannuzzi $^{1}$ and Joseph Michael $^{2}$

${ }^{1}$ L.A. Giannuzzi \& Associates LLC, Fort Myers, Florida, United States, ${ }^{2}$ Sandia National Laboratory, Albuquerque, New Mexico, United States

Focused ion beam (FIB) instruments and other ion beams are routinely used to image materials, prototyping, cross-section materials for 2D or 3D scanning electron microscopy (SEM) and associated techniques and analyses, and to prepare specimens for scanning/transmission electron microscopy (S/TEM). Several prior studies have reported on the observation of ion beam induced transformation of austenitic steels and other metastable austenitic phases bombarded with both light ions such as $\mathrm{He}^{+}$and a variety of heavy ions. In all cases, the transformation is consistent with and dependent on ion species, ion dose, channeling, angle of ion incidence, ion energy, and austenite stability [1-19].

We have revisited the ion-induced austenitic phase transformation in 304L stainless steel and found that the transformation from austenite to ferrite occurs for both $\mathrm{Ga}^{+}$and $\mathrm{Xe}^{+}$ion bombardment subjected to various doses and incidence angles. The transformation mechanism can easily be described using ionsolid interaction theory. As dose increases and the target is subjected to sufficient strain and/or recoil motion, the free energy of formation is significantly reduced when the displaced atoms in the collision cascade nucleate and grow into the more energetically favorable stable ferrite, rather than the original metastable austenite. As shown in figure 1, electron backscatter diffraction (EBSD) shows that ferrite nucleates and grows onto the existing austenite into well-known low energy crystallographic KurdjumovSachs (KS) or Nishiyama-Wassermann (NW) orientation relationships. Since this transformation occurs for different metastable phases and different ions, it is not possible that the transformation mechanism is ferrite stabilization via Ga bombardment as previously suggested [1].

The observation of this transformation has consequences for FIB imaging and milling. Sample preparation of these alloys with ion beam techniques like FIB should be viewed with a great deal of caution and skepticism. The assumption that the sample is representative of the bulk material can only be proven after a careful comparison of the amount of ferrite observed in the prepared sample to the amount of ferrite in the bulk sample.

Sandia National Laboratories is a multimission laboratory managed and operated by National Technology and Engineering Solutions of Sandia, LLC, a wholly owned subsidiary of Honeywell International Inc., for the U.S. Department of Energy's National Nuclear Security Administration under contract DENA0003525.

This paper describes objective technical results and analysis. Any subjective views or opinions that might be expressed in the paper do not necessarily represent the views of the U.S. Department of Energy or the United States Government.

References

[1] Babu, R. I. (2016) Acta Materialia, 120, 391-402.

[2] Bauer, R. J. (2011) Philosophical Magazine, 91(3), 437-457.

[3] Gustiono, D. S. (2004) Materials transactions, 45(1), 65-68.

[4] Hayashi, N. a. (1982) Applied Physics Letters, 41(11), 1100-1101. 
[5] Hayashi, N. S. (1984) Journal of Nuclear Materials, 128, 756-759.

[6] Hayashi, N. S. (1988) Hyperfine Interactions, 42(1-4), 989-992.

[7] Johnson, E. L. (1982) Philosophical Magazine A, 45(5), 803-821.

[8] Jones, H. D. (2016) Materials Characterization, 120, 210-219.

[9] Knipling, K. R. (2010) Materials Characterization, 61(1), 1-6.

[10] Nastasi, M. M. (1996). Ion-solid interactions: fundamentals and applications. New York: Cambridge University Press.

[11] Sakamoto, I. ,. (1990) Journal of applied physics, 68(9), 4508-4512.

[12] Sakamoto, I. H. (1988) Hyperfine Interactions, 42(1-4), 1005-1008.

[13] Sakamoto, I. H. (1991) Journal of nuclear materials, 179, 1053-1056.

[14] Stahl, B. K. (2003) Nuclear Instruments and Methods in Physics Research Section B: Beam Interactions with Materials and Atoms, 211(2), 227-238.

[15] Stroud, P. (1972) Thin solid films, 11(1), 1-26.

[16] Was, G. (1996) Prog Surf Sci, 32, 211-332.

[17] Xie, G. ,. (2000) Journal of nuclear materials, 281(1), 80-83.

[18] Xie, G. .. (2005) Journal of materials research, 20(7), 1751-1757.

[19] Zhao, J. a. (1995) Scripta metallurgica et materialia, 32(10), 1671-1676. 